\title{
ENTROPY ACCELERATION, SHANNON INFORMATION AND SOCIOECONOMICS: QUANTITATIVE EXAMPLES
}

\author{
B.E. LAYTON ${ }^{1}$, S.L NOELL ${ }^{1}$ \& G.A. ORAM JR ${ }^{2}$ \\ ${ }^{1}$ Department of Applied Computing and Engineering Technology, University of Montana, USA. \\ ${ }^{2}$ School of Journalism, University of Montana, USA
}

\begin{abstract}
The primary purpose of this article is to provide additional quantitative examples to the recently presented work [1] wherein a coefficient, $\alpha$, relating the information processing rate, $\dot{I}$, and the entropy production rate, $\dot{S}$, of a system was introduced via $\dot{I}=\alpha \dot{S}$. This expanded manuscript provides further examples of Shannon information content in simple binary systems, additional quantitative examples of the relationship between information and money, and an exposé on how the theory presented in [1] may be viewed in the context of Big History. Specifically, our global civilization is entropicizing our planet at a rate of at least $10^{23}$ times greater than the average universal entropization rate. The cause of this Kurzweilian anthropogenic entropy acceleration is rooted in the Darwinian behavior of individuals as well as entire societies. This article also proposes not only that the 'carnot' and the 'shannon' be introduced as units for entropy and information, respectively, but also that other terms such as 'complexity' be canonized by the sciences in the same manner that terms such as 'work' and 'energy' have been canonized by the engineering disciplines.
\end{abstract}

Keywords: anthropogenic, Big History, contingency, economics, entropy, information, sustainability

\section{INTRODUCTION}

Ever since Richard Feynman first gave a scientific tip of his hat to the engineers Carnot and Shannon for their respective works in thermodynamics and information theory, there has remained an open question regarding the relationship between physical entropy, $S$, with units of $\mathrm{J} / \mathrm{K}$ (joules per kelvin) and abstract information, $I$, with units of $\mathrm{b}$ (bits) [2]. With the blossoming of a global digital age and the mounting manifestations of climate change, a treatment of the relationship between $\dot{I}$ and $\dot{S}$ is warranted. It is also paramount that we consider the socioeconomic factors that can lead to runaway entropy production, especially the potential for obfuscation of information to attain an evolutionary advantage at a societal scale.

Consider the possibility that in our efforts to maintain an orderly environment, we are perpetuating the opposite effect. Since Clausius formalized the work of Carnot into what is now known as the second law of thermodynamics, it is implicit that for any closed system, that entropy must either increase, or at most, remain constant as a function of time [3,4]. There are innumerable ways in which this law manifests itself. But simply stated the second law tells us that, 'Tomorrow the universe will be more disorderly than it is today'. We all have observed the second law at the human scale: coffee cups shatter, books collect dust, teeth decay, oceans become more polluted, etc. Nevertheless, entropy does have physical dimensions, namely energy per unit temperature. In the companion paper and in what follows, we provide quantitative examples of the rate of entropy acceleration, $\ddot{S}$, as well as the relationship between $\dot{S}$ and $\dot{I}$ for several contemporary and historical systems. The general theme of information and entropy flowing through a system is given in Fig. 1. Note that energy flowing through the system is implied.

One of the primary motivations for using entropy generation rate $\left(\mathrm{J} \mathrm{s}^{-1} \mathrm{~K}^{-1}\right)$ rather than specific power $\left(\mathrm{J} \mathrm{s}^{-1} \mathrm{~kg}^{-1}\right)$ [5,6] as a universal metric for 'complexity' or 'sustainability' is to examine how individuals as well as entire societies organize their information systems to build physical power systems (machines) to either deentropicize their own immediate environments or to entropicize the 


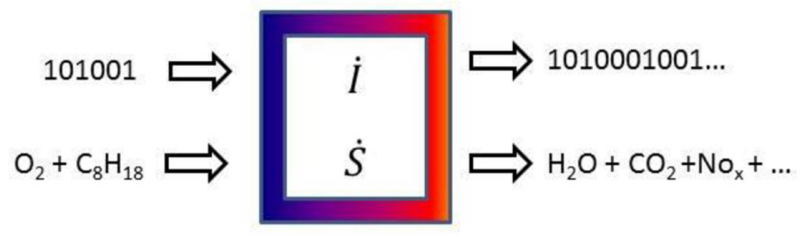

Figure 1: Basic model presented in the article, with information, and low-entropy material flowing into a system coupled with information and high entropy material flowing out. Survival of a given system may thus be defined by a system's ability to process information and shed entropy to avoid an internal entropic event.

environments of other individuals or societies, i.e. individuals within one society may collaborate share information - to build a sewer system to avoid local water contamination, to build multifamily housing to share and save resources, to form a cooperative healthcare plan, etc. On the other hand, individuals within a society may share information to build a destructive device such as a weapon to destroy (entropicize) a competitor. This competitor could be an individual or a society competing to acquire physical objects such as natural or manufactured resources or memetic structures such as trade secrets or belief systems. While using specific power as a metric for typifying systems or societies is useful, it misses the subtleties inherent in the second law; it does not account for environmental degradation; and it remains silent on the notion that information flowing through power systems can be used to direct or dissipate waste energy.

In physical terms, the second law tells us that the universe is constantly 'reverse funneling' energy from dense, easily accessible forms, e.g. gravitational, kinetic, chemical, nuclear, etc. into diffuse, less accessible forms: e.g. radiation, acoustic, thermal, etc. On the other hand, we know that if we wish to 'unmix' diffuse forms, 'primary energy' [7] must be provided [8], thus begetting additional entropy.

Throughout this treatment and the accompanying conference proceedings [1], several humanscale examples are presented to quantify entropy and information generation rates of common human activities. However, before examples can be given, a thorough quantitative exploration of entropy at scales both above and below the human scale is required. This is followed by a discussion on information content of various physical systems, and finally by the introduction of an 'informational fitness' coefficient that relates entropy production rates to information generation rates. In the concluding section of the article, we also discuss how information is leveraged to generate financial wealth.

\section{ENTROPY AND ENERGY AT THE LARGE SCALE}

Human civilization currently sits at approximately $125^{\text {th }}$ of a Kardashev Type I society. Equivalently, we are at $125^{\text {th }}$ of Diamond's photosynthetic ceiling. However, the chemical energy we are currently exploiting is being extracted from an extraordinarily ancient 'solar bank'.

A financial analogy illustrates our energy deposition-to-withdrawal rate. If someone had a rich uncle deposit one dollar per minute into her trust fund for 100 consecutive years, she would have approximately $\$ 50 \mathrm{M}$ on her $100^{\text {th }}$ birthday. Analogously, since our hydrocarbon extraction rate is ten million times greater than the deposition rate (300:3,000,000,000 years) [9], this implies that withdrawals from the fund would not begin until the heiress was 99 years, 364 days, $23 \mathrm{~h}$ and 55 min old. We are thus midway into a volatile 5-min hydrocarbon-fueled party with $2 \frac{1}{2}$ min until last call [10]. 


\subsection{Entropy generation rate of the sun}

As recently demonstrated [1] humans entropicize their environment at a rate of $1.80 \times 10^{23}$ times greater than the average 'background' entropy generation rate of the universe. This is a direct result of the fact that $100 \%$ of our 500+ exajoule-per-year technological diet becomes thermal. Normalizing both the universal and the terrestrial rates of entropy generation by their respective volumes results in $\dot{S}_{\text {universe, } \mathrm{V}}=2.14 \times 10^{-32} \mathrm{~W} \mathrm{~K}^{-1} \mathrm{~m}^{-3}$ and $\dot{S}_{\text {earth,V }}=4.33 \times 10^{-9} \mathrm{~W} \mathrm{~K}^{-1} \mathrm{~m}^{-3}$. For further reading on the universal entropy generation rate, see [11].

Note the units on $\dot{S}_{\mathrm{V}}$. This metric is the rate at which energy becomes inaccessible to do work. Even though living systems [12], and indeed entire celestial bodies [13], as demonstrated in following paragraph can reduce their entropy locally, $\dot{S}_{\mathrm{V}}$ will presumably always be non-negative when considered on a sufficiently large scale, e.g. [14]. Note also that with the addition of each energy technology system such as a coal-fired power plant, nuclear power plant, wind farm, etc., that the rate at which $\dot{S}_{\text {earth, V }}$ is increasing is also positive, i.e. $\ddot{S}_{\text {earth, }}>0$. The fundamental question is whether or not this accelerating rate of disorder will cause increased scrambles to contain or mitigate its effects, thus leading to a sort of runaway chaotic state that necessitates some sort of naturally mandated de-escalation or de-evolution away from energy-intensive technologies, e.g. [15].

\subsection{Entropy generation rate of a pair of planets}

Two planets of equal size and mass but at two different temperatures quantitatively demonstrate the non-conservative nature of the second law [13]. If planet A is at $289 \mathrm{~K}$ and planet $\mathrm{B}$ is at $283 \mathrm{~K}$, then spontaneous energy transfer will occur from planet A to planet B. If radiant energy transfer occurs at a rate of $2330 \mathrm{~W} / \mathrm{kg}$, the mass-specific entropy generation rate of planet $\mathrm{A}$ is

$$
\Delta \dot{S}_{\mathrm{A}}=\dot{q} / T_{\mathrm{A}}=-2330 \mathrm{~W} \mathrm{~kg}^{-1} / 289 \mathrm{~K}=-8.062 \mathrm{~W} \mathrm{~kg}^{-1} \mathrm{~K}^{-1},
$$

whereas the entropy generation rate of planet $\mathrm{B}$ is

$$
\Delta \dot{S}_{\mathrm{B}}=\dot{q} / T_{\mathrm{B}}=-2330 \mathrm{~W} \mathrm{~kg}-1 / 283 \mathrm{~K}=-8.233 \mathrm{~W} \mathrm{~kg}^{-1} \mathrm{~K}^{-1},
$$

and thus the entropy production rate, or the rate at which the energy transfer from planet A to planet $\mathrm{B}$ entropicizes the universe on a per mass basis is

$$
\Delta \dot{S}_{\mathrm{U}}=\Delta \dot{S}_{\mathrm{A}}+\Delta \dot{S}_{\mathrm{B}}=-8.062 \mathrm{~W} \mathrm{~kg}^{-1} \mathrm{~K}^{-1}+8.233 \mathrm{~W} \mathrm{~kg}^{-1} \mathrm{~K}^{-1}=0.171 \mathrm{~W} \mathrm{~kg}^{-1} \mathrm{~K}^{-1} .
$$

For our own planet, is there a way to subvert this 'entropy cascade' currently being propagated by our earthbound heat engines? Maybe. One particular emerging technology in particular could help shed terrestrial thermal energy by radiating within a bandwidth invisible to the molecules in the atmosphere [16]. One could imagine such a technology expanding across vast expanses of the earth that are currently merely acting as heat sinks.

\section{ENTROPY AT THE SMALL SCALE}

There is also a means of quantifying thermodynamic entropy on a classical (non-quantum) microscopic scale. For example, the Gibbs entropy [17] of a system is defined as

$$
S=-k_{\mathrm{B}} \Sigma_{\mathrm{i}} p_{\mathrm{i}} \ln p_{\mathrm{i}}
$$

where $k_{\mathrm{B}}$ is Boltzmann's constant and $p_{\mathrm{i}}$ is the probability of finding a particle within the system under consideration occupying a particular energy level. This expression is valid for purely 
thermodynamic systems, not physical systems in general, and is only one of several generally recognized definitions [17]. However, if one were to develop an exhaustive set of independent physical variables for a physical system, in principal, the same general statistical approach could be taken to establish an entropy metric for multiphase multicomponent systems.

It follows directly from (4) and from the first law that for a system where mass is conserved, i.e. the atmosphere, that if the temperature increases as is the case with combustion engines, urban heat islands, etc., that more energy per particle simply implies that on average the molecules in the atmosphere are on average, moving faster as time proceeds.

Recall that the working definition of the second law states that entropy cannot decrease for any closed system, i.e. any system from which energy neither leaves nor enters. As Schrödinger observed in 1944 [12], organisms consume energy to locally and temporarily reduce their own entropy. Even though an organism may reduce its own entropy, it does not violate the second law, because living systems are open. Place this organism inside a sealed vessel [closed system] with no access to nutrients or any means of eliminating waste, and it will eventually die, having become a victim of entropic forces.

The earth itself is also an open thermodynamic system, with photosynthetic energy coming in and low-grade thermal radiation leaving. All modern and past societies live downstream of this photosynthetic energy. It is instructive to note that without advanced mining, the Roman Empire could only expand as quickly as they could access recently stored photosynthetic energy. This resulted in the exhaustion of all available resources and thus a point of unsustainability and collapse [18]. With command over fire, but no fossil fuel or nuclear technologies, they were unable to reach the quality of life and population densities that we experience today.

Looking at our own sustainability as a global society that relies predominantly on the burning of fossil fuels, with carbon dioxide preventing a significant fraction of our waste heat from leaving our planet, are we not running the risk of accelerating our own demise by basking in our own entropy? A growing number of people have indeed been designated as 'climate change refugees', by authors such as Hollifield et al. [19] and Sachs [20].

\section{INFORMATION}

Information is dimensionless and has as its unit, the 'bit': $0=$ off and $1=$ on. Simply stated, the amount of information a given message carries is merely the minimum binary word length to convey a message between two entities. The two entities could be two people talking, a person listening to the radio, the mating call of a pair of whales or a computer receiving a software update. Thus, any noise in the signal or in the medium detracts from the fidelity of the transmission. Information content $I$ is defined formally as

$$
I=-\Sigma p_{\mathrm{i}} \ln _{2} p_{\mathrm{i}}
$$

where $p_{\mathrm{i}}$ is the probability of a particular event occurring.

The proper conveyance of information depends on the type of machine (or organism) sending the message and the type of machine (or organism) receiving the message. For example, if two people are to have an 'efficient' conversation, they must understand the same language. For a person to hear what's on the radio, the radio must be well tuned with little 'noise' on its frequency. For two whales, the sound waves must be properly directed and be interference free from sonar. And in the case of the computer, the physical media (CD ROM, hard drive, integrated circuit, etc.) must be in proper working condition, and the error-checking algorithms must be flawless. 


\subsection{Key distinction between entropy and information}

Notice the similarity of eqns (4) to (5). Both $S$ and $I$ are scalars and both contain the product of a probability and its logarithm. The key difference is that information is dimensionless, and [physical] entropy has dimensions of energy per unit temperature. The word 'physical' is in brackets for two reasons: first, to emphasize that entropy is just as physical as mass, space time, charge, etc., and second, to stress that entropy has dimensions of energy per unit temperature. More subtly, since entropy has dimensions of energy per temperature and temperature has units of energy per particle, in this sense, entropy with the same dimensions as Boltzmann's constant, shares the same dimensions as its system of particles it quantifies.

4.1.1 Key distinction between entropy and information: a 'carnot' and a 'shannon'

The formal proposal for an internationally recognized unit of entropy is long overdue. A reasonable SI unit is J/K, the 'carnot'. In fact, a major motivation for this article was to address Feynman's 1963 claim that only two engineers in history have ever contributed to the scientific body of knowledge: Carnot and Shannon, and that their theories were related [2]. Perhaps, a 'shannon' could represent the number of bits required to embody his life's work [21]. Let us give these engineers their due.

\subsection{Information and entropy examples}

In any information system, each character used in the language/code being transmitted can be assigned a level of importance in terms of the integrity of the message and thus the likelihood of the speaker/transmitter being understood by the listener/receiver. For example, when transmitting driving directions via a series of symbols, confusing 'turn east' with 'turn west' could have serious consequences. This incorrect message occurring would require that 'ea' be replaced with 'we'. Thus, only 'ea' and 'we' contain information, whereas 'st' contains no information beyond what is required for communication in general.

Information theory as originally published by Shannon [22] is more subtle than discussed above in that the order of the characters also contains a measureable probability and thus measureable amounts of information. For example, the sequence 'th' is more likely to occur than the sequence 'ht'; therefore, 'ht' contains more information because it is rarer. For the purposes of this article, we are merely concerned with the minimum number of bits required to reproduce a given artifact, meme, idea, etc. Bits only have utility insofar as they can be either transmitted as discussed in the opening paragraphs of this section.

4.2.1 Information content of the Mona Lisa versus a Boeing 787

For the time being, we use the binary unit to quantify complexity: the complexity of a fully compressed binary string is equal to the minimum number of bits required to faithfully reproduce it.) We will use this definition to compare two well-known artifacts. The Mona Lisa has been photographed numerous times and has thus already been digitized. Undoubtedly, none of the various photographic media used to represent the painting possess a resolution great enough to physically reproduce La Gioconda molecule by molecule. The complexity for the airplane is likely well above the terabit range and the painting is likely another dozen orders of magnitude greater (assuming that all Boeing 787s are identical). Of course, due to manufacturing and material inconsistencies, each plane is unique. Accounting for non-identicality would raise the complexity many orders of magnitude (based simply on the number of atoms in the plane). One approach to 'informatize' the Mona 
Lisa would be to give the type and location of all its atoms. With a mass on the order of $200 \mathrm{~g}$, this yields approximately $200 \mathrm{~g} \times 0.0833 \mathrm{~mol} \mathrm{~g}^{-1} \times 6.022 \times 10^{23}$ atoms $\mathrm{mol}^{-1} \approx 1 \times 10^{25}$ atoms. Allowing for 64 different atom types ( 6 bits per type) and 90 bits per location results in $5.7 \times 10^{30}$ bits for a full recreation of the Mona Lisa.

Now that this framework of how to quantify information in an artifact has been established, let us develop a more formal relationship between entropy and information by examining some simple systems where information is easily quantifiable.

\subsection{Simple two-state information systems}

Consider a system consisting of two particles, both of which can have two states, such as a pair of coins. This two-coin system must occupy one of four states: HH, TT, HT or HT. If we have $\mathrm{p}_{\mathrm{H}}=$ $p_{\mathrm{T}}=1 / 2$, then the information content of each of these four states is identical, and maximized, because from (5),

$$
I=-\left\{\left[1 / 2 \log _{2}(1 / 2)+1 / 2 \log _{2}(1 / 2)\right]+\left[1 / 2 \log _{2}(1 / 2)+1 / 2 \log _{2}(1 / 2)\right]\right\}=2 .
$$

In other words, each single flip of the coin contains one bit of information, and all four states that result from consecutive flips contain two bits of information.

On the other hand, if $\mathrm{p}_{\mathrm{H}}=0.1$ and $p_{\mathrm{T}}=0.9$, then $I_{\mathrm{HH}}=0.664, I_{\mathrm{HT}}=I_{\mathrm{TH}}=0.469$ and $I_{\mathrm{TT}}=0.274$. In other words, the more likely event contains less information than the less likely one. Moreover, a system where the probability of complimentary states is unequal is less capable of carrying information than a system where both states are equally likely. In this lopsided case, the total information carried by the unequal probabilities is 0.938 as opposed to 2 :

$$
I=-\left\{\left[0.1 \log _{2}(0.1)+0.9 \log _{2}(0.9)\right]+\left[0.1 \log _{2}(0.1)+0.9 \log _{2}(0.9)\right]\right\}=0.938 .
$$

A list of the information content of other lopsided binary systems is given in Table 1.

Table 1: Summary of information content in various symbolic systems: (a) a two-state system,

\begin{tabular}{|c|c|c|c|c|c|c|}
\hline (a) & & & (b) & & (c) & \\
\hline$p_{1} / p_{2}(\%)$ & $I_{1}$ & $I_{2}$ & $p_{1} / p_{2} / p_{3}(\%)$ & $I_{1}$ & $p_{1} / p_{2} / p_{3} / p_{4}(\%)$ & $I_{1}$ \\
\hline $50 / 50$ & 1 & 2 & $3 \overline{3} / 3 \overline{3} / 3 \overline{3}$ & 1.585 & $25 / 25 / 25 / 25$ & 2 \\
\hline $60 / 40$ & 0.971 & 1.942 & $25 / 25 / 50$ & 1.5 & $10 / 10 / 10 / 70$ & 1.357 \\
\hline $75 / 25$ & 0.811 & 1.623 & $10 / 10 / 80$ & 0.922 & $1 / 1 / 1 / 97$ & 0.242 \\
\hline $90 / 10$ & 0.469 & 0.938 & $1 / 1 / 98$ & 0.161 & 0/0/0/100 & 0 \\
\hline $99 / 1$ & 0.081 & 0.162 & 0/0/100 & 0 & & \\
\hline $99.9 / 0.1$ & 0.011 & 0.023 & & & & \\
\hline $100 / 0$ & 0 & 0 & & & & \\
\hline
\end{tabular}
(b) a three-state system and (c) a four-state system.

$p_{\mathrm{i}}$ represents the probability of the $i$ th state, $I_{1}$ represents the information content of a string of independent characters one character long and $I_{2}$ represents the information content of a string two characters long. In (a) the characters are independent: the state of one character does not affect the state of the other. 
The same formulae that were applied to generating the data in Table 1 can be applied to a language system. For example, the letter 'e' contains less information than the letter 'q' because 'e' occurs more frequently. Thus, if we were to decide to rewrite our dictionaries so that our various languages would be more 'efficient' at carrying information, we would likely begin by eliminating letters such as the superfluous ' $u$ ' after ' $q$ '. Note that this would be $100 \%$ valid if all instances of q were followed by u. Though rare, exceptions familiar to Scrabble aficionados such as 'qat' and 'sheqel' do exist.

Note that we have explored quantitative examples of entropy production as well as information content, we must move onto our goal of producing a more formal relationship between $\dot{S}$ and $\dot{I}$, especially as they relate to the contingencies inherent in socioeconomics.

\section{INFORMATION AND ENTROPY}

From Lin [23] 'For information-theoretic entropy, if one uses entropy and information interchangeably, ... we cannot make meaningful intellectual discussion'. He continues with the encouraging words:

However, the messy and confusing situation regarding entropy-related studies has provided opportunities for us: clearly, there are still many very interesting studies to pursue. For instance, one immediate task is to investigate whether the information-theoretic entropy and the thermodynamic entropy are compatible or not compatible, i.e. (i) whether both need to satisfy a definition of 'information is related to entropy loss', (ii) what relation the information-theoretic entropy has to the second law of thermodynamics and (iii) whether there is any correlation between informationtheoretic entropy and thermodynamic entropy.

Reading this in 2013 after having contributed to the field [24-27] leaves a clear and encouraging path once the acknowledgment has been made that information is dimensionless and is measured in bits, and entropy is physical and is expressed as joules per kelvin. Armed with this knowledge, we will see that each of Lin's three questions may be answered in the affirmative.

Every system, living or non-living, must abide by the second law of thermodynamics throughout its lifetime. In other words, every system, whether living or non-living contributes to leaving the universe more entropic than it would have been otherwise. This assumes that all Type II and greater machines act independently. In reality, this is not the case, since some subsets of 'high entropy' humans, which are themselves Type IV machines have used Type I and Type II machines to kill other subsets of 'low entropy' humans [28]. Every system, living or non-living, also requires a finite amount of information to reproduce or be reproduced. (Only Type IV machines, are capable of self-reproduction: [24, 29].) Therefore, every system, living or non-living, serves as an information source, an information sink, or both, as well as an entropy source, an entropy sink or both (Fig. 1).

\subsection{The Google car and 'informational fitness'}

To further address Lin's three questions, how can we quantitatively illustrate the relationship between information and entropy? More specifically, how might having accurate information allow one to forgo the generation of 'excessive' entropy? Consider Google Maps [30]. The reader may have seen a Google car driving through her neighborhood generating entropy with its internal combustion engine at a rate of $\dot{S}_{\text {Google car }}=50-100 \mathrm{~W} \mathrm{~K}^{-1}$ and 'informatizing' the environment at a rate of $\dot{I}_{\text {Google car }}=1-10 \mathrm{~Gb} \mathrm{~s}^{-1}$. We thus assign a coefficient, $\alpha$, that acts as a scalar between information generation and entropy generation:

$$
\alpha=\dot{I} / \dot{S} .
$$


This scalar $\alpha$ may be considered as a 'sustainability coefficient' that measures the 'informational fitness' of a given system, which essentially quantifies how capable the system is of storing new information as it generates entropy. Using the entropy generation rate and the information processing rates we estimated above for the Google car, we arrive at $\alpha_{\text {Google car }} \approx 10^{9} \mathrm{~b} \mathrm{~s}^{-1} / 10^{2} \mathrm{~W} \mathrm{~K}^{-1}=10^{7} \mathrm{~b} \mathrm{~K} \mathrm{~J}^{-1}$. Or in words, the informational fitness metric of the Google car is 10,000,000 bit-kelvins per joule. To take this one step further, since the fundamental dimension of temperature is energy per particle, $\alpha_{\text {Google car }} \approx 10^{7} \mathrm{~b}$ particle ${ }^{-1}$. In this case, the particle is simply the car. But assuming approximately $10^{29}$ atoms in the car yields $\alpha_{\text {Google car }} \approx 10^{-22} \mathrm{~b}$ atom $^{-1}$.

\subsection{A computer virus example}

Consider a computer virus. Fundamentally, it is a very simple computer program, consisting of as few as 1000 bytes ( $~ 8000$ bits). But what is the virus's informational fitness? There are entire divisions of companies devoted to outwitting computer viruses. So arguably, all of the entropy generated by all of the employees in the anti-virus division is being devoted to 'killing' the virus. Let us say that it takes the effort of 10 people 1 month to write an effective antivirus code. If those ten people are functioning at the average energy throughput of a North American $(1200 \mathrm{~kW})$, then the informational fitness of the computer virus is

$$
\alpha_{\text {virus }}=8000 \mathrm{bits} /(10 \times 1200 \mathrm{~W} \times 2,592,000 \mathrm{~s} / 300 \mathrm{~K})=7.7 \times 10^{-6} \mathrm{~b} \mathrm{~K} \mathrm{~J}^{-1} .
$$

How is this to be interpreted in comparison to the fitness of the Google car in light of the estimation that $\alpha_{\text {Google car }}=10^{12} \alpha_{\text {virus }}$ ? Does this mean that the Google car is a trillion times more fit than the virus? No. The way to interpret this example is to realize that a tremendous amount of energy had to be expended and entropy generated to get the computer programmers to and from their offices, and that this mode of 'information warfare' is relatively inefficient as viewed from our sustainability coefficient or informational fitness perspective.

There are many other perspectives from which this scenario can be evaluated such as measuring the amount of original code (information) that the programmers generated to 'defeat' the virus. Perhaps more interesting to consider though is the number of other computers that may have avoided infection as a result of the programmers' efforts. Defeating the virus before it infected numerous other computers avoided the eventual entropy generation that would have resulted from having to take the infected computers to the repair shop, etc.

\subsection{A home energy monitoring example}

As another practical example of the relationship between an easily quantifiable information stream and an easily quantifiable entropy stream, consider some of the remote home energy monitoring systems that are now on the market. An operator of this system might get 20 channels or information streams, giving such data as the status of the lighting of 10 circuits in the house, each of which is binary, i.e. 'on' or 'off' as well as the temperature and status of the temperature controls for each of five zones. The lighting circuits contain 10 bits of information, e.g. 'on, off, off, on, on, off, off, off, off, on' or ' $1,0,0,1,1,0,0,0,0,1$ '. The user could then choose to turn all of the lights off: ' 0 , $0,0,0,0,0,0,0,0,0$ '. If the data for the lights were sent every minute, then the information rate is $\dot{I}=0.166 \mathrm{~b} \mathrm{~s}^{-1}$. If we assume that each of the four lights that were left on were $50 \mathrm{~W}$ bulbs, and that their primary energy source is coal with an efficiency of $33.3 \%$, then the energy consumption rate of the four bulbs is $3 \times 4 \times 50 \mathrm{~W}=600 \mathrm{~W}$, and the entropy generation rate is thus $\dot{S}=600 \mathrm{~W} / 288 \mathrm{~K}=2.08 \mathrm{~W} / \mathrm{K}$ and thus $a$ of the system prior to switching off the four lights 
is $\alpha_{\text {lights_on }}^{-1}=\dot{S} / \dot{I}=(2.08 \mathrm{~W} / \mathrm{K}) /\left(0.166 \mathrm{~b} \mathrm{~s}^{-1}\right)=12 \mathrm{~J} \mathrm{~K}^{-1} \mathrm{~b}^{-1}$. If we assume that the monitoring system itself consumes energy at a rate of $10 \mathrm{~W}$ and that all of its energy comes from a renewable energy system, then after the lights are off, $\alpha^{-1}{ }_{\text {lts off }}=0.21 \mathrm{~J} \mathrm{~K}^{-1} \mathrm{~b}^{-1}$. And thus our informational fitness for both of the two states is given by their respective 'sustainability coefficients' $\alpha_{\text {lights on }}=0.083 \mathrm{~b} \mathrm{~K} \mathrm{~J}^{-1}$ and $\alpha_{\text {lights_off }}=4.8 \mathrm{~b} \mathrm{~K} \mathrm{~J}^{-1}$. In other words, after the lights have been turned off, $5 \overline{7}$ times as many bits per joule are being transmitted at a given temperature and thus the house with the lighting technology is making a smaller contribution to 'global entropization'.

\subsection{Other information-entropy examples}

A similar analysis could be performed for nearly any system: using a GPS to navigate through an unfamiliar town vs. using an outdated map, making a decision whether or not to invade a country with our without information regarding the local customs, engineering the landing gear for a spacecraft with or without information on the physical properties of the surface, etc. Similar analyses could also be applied in the realm of biology: whether or not to perform cell division or reproduction based upon the availability of nutrients or resources, whether or not to move towards a nutrient source with or without the information on local predators, etc.

As we have seen, not all societies or individuals generate entropy at the same rate [27]. Consider the entropy generation rate of riding a bicycle at $20 \mathrm{mph}$ vs. driving a gasoline-powered car at the same speed [31]. Both serve the same purpose: to deliver the passenger to his destination, but the automobile, occupying a state much further from equilibrium, e.g. [32], generates entropy more quickly. It is also noteworthy that embodied information of a bicycle is typically much less than the embodied information of a modern automobile.

Let us now explore entropy and information flow in living systems. Genet [33] in his book Humanity states: 'The first era would be life before the DNA encoding of information, an era when information and metabolism were one and the same thing'. This he attributed to Dyson [34]. This statement may be interpreted to mean that since there was no dedicated and firmly established molecule for storing information, any molecule that might have been vying for that role was just as likely to be consumed for its energy, rather than preserved for its ability to offer advice to future generations. A similar analogy might be drawn between the relative value of a biological specimen preserved in a coal or peat reserve: is it more valuable to combust (entropicize) it or to analyze and record (informatize) its molecular structure in order to place it along the path of the Ancestor's Tale [35]?

As mentioned in the Introduction in Chaisson's chapter in Cosmos and Culture [36], he argues that an energy metric plays a role in evolution. He corroborates the observation that natural selection is indeed not really selection, but rather a set of things that are left over after other entities or systems have been eliminated. While this laissez-faire view of evolution is more than likely correct in the sense that there is no overarching goal or meaning to evolution, it would be difficult to defend the position that the organisms that are now extinct are in some way 'fitter' than those that are now living, or that societies that have succumbed to others are somehow more fit than their conquerors. The only argument that remains in this ongoing debate is whether the capacity to destroy is an appropriate fitness metric.

As predicted by Toffler in his book, The Third Wave, nearly 30 years ago, we are now fully immersed in the Information Age [37]. One upshot of this new reality is that people with better access to information of consequence such as what the weather be next week, which stocks are likely to rise in value, what the structure of a virus is, whether or not there is a cancerous tumor present in one's brain, whether or not someone trying to break into your house, etc. are fitter, and thus more 
likely to avoid negative (entropicizing) events. Thus, one's ability to avoid self-entropization is contingent upon one's access to information. So in addition to our physical and mental fitness, both of which are biological, we must now include 'information technology fitness' as a new metric for measuring an individual human's fitness level.

\section{INFORMATION, ENTROPY, GAME THEORY AND CONTINGENCIES}

How might misinformation work at the societal level? One recent famous example was Colin Powell's famous statements at the United Nations that led to hundreds of thousands of Iraqi deaths and a comparable number of American families who are now dealing with deceased relatives, chronic physical injuries and ongoing mental instability in the name of fossil-fuel-based energy security. This political charade continues as we pretend that smokestacks, nuclear reactors and automobile exhaust pipes do not heat the atmosphere. This reality of the potency of misinformation regarding the relationship between greenhouse gas emissions and climate change continues to abound in the mass media.

Let us turn now to another socioeconomic example by examining the democratic political process through the lens of information and entropy. If the goal of the politician is to stay in office, she will need to convince more than half of the population that she is 'on their side' in regard to an issue or set of issues. The politician may even need to lie to herself to get elected. But regardless of the politician's personal viewpoint on a particular issue, the stance taken on each of the myriad of political issues will affect voter decisions. Let us dissect the information contained in two opposing platforms as an example: 'We should mine uranium' vs. 'We should not mine uranium' (Fig. 2). Since our goal is unification of the information landscape with the entropy landscape, let us first turn these two statements, each of which the next election is likely to be contingent upon, into information with an ASCII to binary converter. In binary, they look like so:

Of course, these two statements must then be evaluated by the public, each individual must process them and then make his or her own binary decision as to whom to vote for. The numbers are tallied, and then another binary decision is made: candidate A or candidate B. The 32-bit difference between the two statements and their alignment with the belief system of the voting citizenry will thus determine the resulting entropy related to mining uranium or not.

Incidentally, mining uranium to make electricity may be a better alternative to mining coal from an environmental entropization perspective since the primary emission is heat rather than carbon dioxide. Presently, it is not clear whether a nuclear power plant or a coal-fired power plant generates entropy faster than it slows the rate of human deentropization, but based upon the recent Fukushima disaster, one could argue that in some instances, coal is the lesser of two environmental poisons. Perhaps, with further study and measurement of the sustainability coefficient of these competing technologies, a consensus might be achieved to select the most appropriate technology.

$$
\begin{aligned}
& 0101011101100101 \underline{00100000} 01110011 \\
& 01101000011011110111010101101100 \\
& 01100100 \underline{0100000} 0110110101101001 \\
& 0110111001100101 \underline{00100000} 01110101 \\
& 01110010011000010110111001101001 \\
& 0111010101101101
\end{aligned}
$$

$0101011101100101 \underline{00100000} 01110011$ 01101000011011110111010101101100 $01100100 \underline{00100000} 0110111001101111$ $01110100 \underline{00100000} 0110110101101001$ $0110111001100101 \underline{00100000} 01110101$ 01110010011000010110111001101001 0111010101101101

Figure 2: ASCII to binary codes for 'we should mine uranium' and 'we should not mine uranium'. The spaces have been underlined, and the word 'not' has been emboldened. 


\section{ENTROPY AND INFORMATION IN THE CONTEXT OF SOCIOECONOMICS}

\subsection{Complexity and entropy}

In David Christian's TED talk [38] he asserts that 'complexity is the antithesis of entropy'. As with the misinformative justification for invading the Middle East, complexity implies that a system that appears inexplicable at first, actually has an underlying truth and thus order, however, convoluted it may be. As there has yet to have been a consensus metric or dimension for complexity, we will continue to use the bit: something that is more complex requires more bits to represent it. Nevertheless, declaring something to be 'complex' implies that there is hope in revealing the underlying order in a system that at first seems random. This has always been the overarching goal of science: to understand the universe via a small set of rules, e.g. $f=m a, E=m c^{2}, x^{\prime}=x(\alpha-\beta y) ; y^{\prime}=-y(\gamma-\delta x)$, etc.

Is there a 'unified equation' of contingency and socioeconomics lurking on the horizon? Since the overarching goal of science is to make predictions, presumably socioeconomics will eventually 'catch up' to the other sciences that have become quantitative. Any such equation or system of equations would of course capture the underlying truths of nature as well as the vagaries of human nature that both reveal the underlying mathematical beauty of the universe and create the seemingly random contingencies through which socioeconomic events are created.

There are several books that have begun to at least address this question in a qualitative sense. In Robert Wright's Moral Animal, the author states that there are a few metrics to male-female relationships. Many of these may be regarded as Nash problems [39], wherein the solution space is complex and often difficult to navigate in tandem [40]. Exploring one's own space and finding the boundaries often lead to conflicting spheres of interest and the risk of disjunction. In any case, 'the moral animal' is always 'gaming' the system either wittingly or unwittingly for self-benefit.

\subsection{Socioeconomics, the economy and the environment}

In Beinhocker's Origin of Wealth [41], the author states that 'there are three modes of wealth generation'. To paraphrase, these are 'lack of goods, lack of knowledge or lack of money'. A fourth should be added: 'Lack of time'. He also states that irreversibility is inherent in wealth generation: one cannot burn the same lump of coal or absorb the same photon twice. But he does neglect to mention repeatability: the same power plant can burn another lump of coal, and the solar panel can absorb another photon just as Bernie Madoff found multiple clients.

In Brandenburg and Paxon's Dead Mars Dying Earth [42], the authors make the poignant statements that: 'The environment is the economy'. This statement contains the same fallacy as does the statement 'entropy is information'. Nevertheless, Brandenburg and Paxon go on to say 'The economy is simply a way of describing one dimension of the environment'. While none of the authors above use any mathematics to support their statements in their layman's texts, if we are to move forward towards sustainability rather than economic and environmental collapse, we should heed Brandenburg and Paxon where they state:

We must give up the illusion that it [the economy] has a separate and competing existence. It doesn't. No environment, no economy.

They further elucidate the statement with 'We created economies as a way of being more flexible and expeditious in how we relate to the environment: steel from forges can be turned into plows to sell to farmers, to grow food to feed steelworkers... In fact, we now depend upon "the economy" in 
exactly the same way that we depend upon the environment - for food, shelter and every other material good we purchase or consume - but the economy does not have an independent existence. It is a co-existing dimension of the environment'.

That the environment and the economy are now inextricably linked is the wake-up call that all technologists must grapple with. But how do we 'measure the environment?' How do we 'measure the economy?' The first thing to face is the fact that neither are scalars. In fact, it is likely that neither are vectors or even some lower order tensors. It is likely that the environment and the economy can take on as many dimensions as our minds and models are capable of conceiving.

On the environmental side, we may never know how complex the environment writ large, i.e. the universe, truly is and thus may never have a unified theory of everything. Many talented physicists are working on this of course, struggling to find that perfect balance on Occam's razor.

On the economic side, since money and its various contrived facets are merely agreed upon units to measure everything from gold bars, to land, to ideas, to time, etc., we collectively and often unwittingly 'decide' how complex to make our economy on a daily basis.

\subsection{Information and money}

With these notions of what the environment and the economy are in mind, we must now determine what the system for describing their relationship should be. For this, we must turn to information itself. It is information that we gather when we measure $\mathrm{CO}_{2}$ levels or temperature. It is information we gather when we count the number of penguins or Ebola viruses. It is information we gather when we observe the sun's spectrum, etc. When it comes to measuring the economy, it is information that we gather when we measure the growing income gap. It is information that we gather when we evaluate competing insurance policies or retirement plans. What we can gather from this is that eqn (8) is certainly not the final answer to our question, but merely a one-dimensional expression of a larger truth. Nevertheless, as a starting point, we can begin with

$$
\dot{m}=\beta \Delta \dot{I}
$$

or in words, the rate at which money is made is proportional to an information gap. The individual with greater information at his or her disposal will be able to make money faster than the individual with less information in a given realm: business, law, engineering, education, real estate, etc.

If we are to achieve a more sustainable balance between our economies and our environment, it is critical that information about the environmental consequences of our current technologies be brought to as many people as possible. To a large extent Neil deGrasse Tyson makes an attempt at this in episodes 11 and 12 of season 1 of Cosmos: in Episode 11 he states that the economy acts on a much shorter timescale than the environment. In Episode 12, he points out the economic and political contingencies that led to the demise of two technologies that could have led us to very different current environmental realities: 1896 World's Fair solar fridge and 1915 solar irrigation system. Had it not been for a quick and dirty race to the bottom for cheap energy, we might not have the mounting greenhouse gas problems that we are experiencing today.

\subsection{Socioeconomics and Big History}

It now behooves us to look at recent histories of our various civilizations in an effort to learn from our recent past. For example, each human in each of Christian's three major Eras: Foraging, Agrarian and Modern, certainly had his or her own capacity to process energy as well as his or her own capacity to process information. To an extent and in general, humans who had the greatest capacity to 


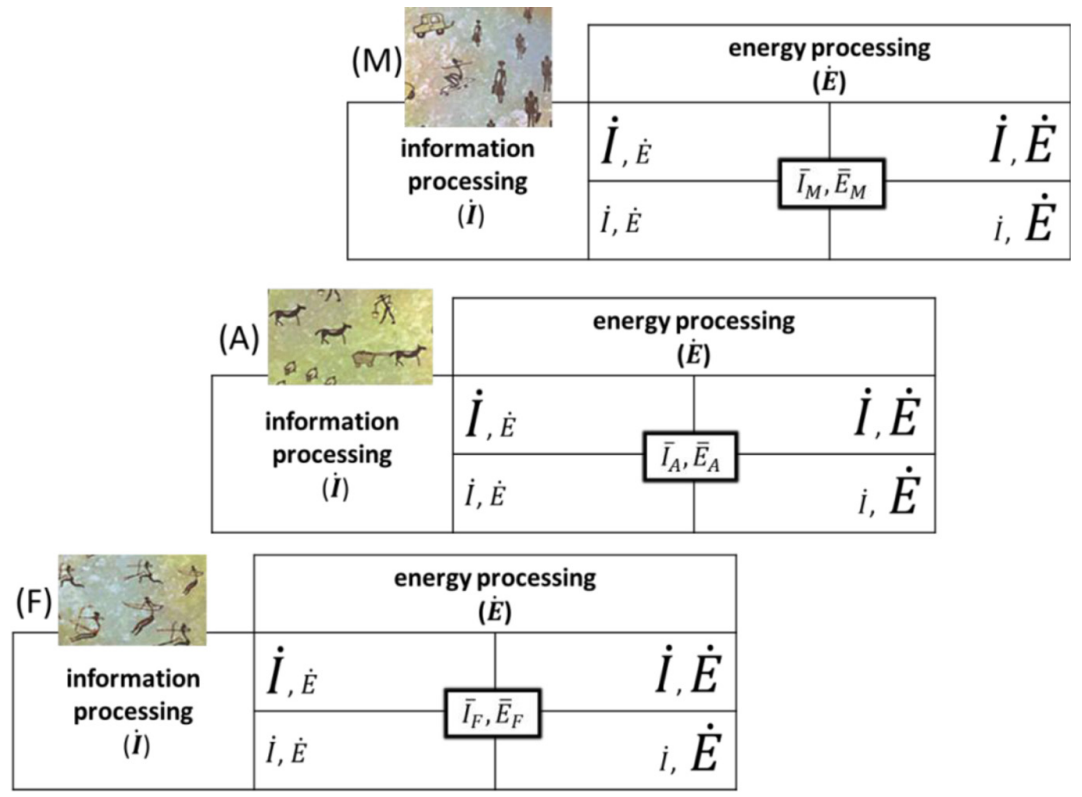

Figure 3: A framework for comparing Christian's three major eras: foraging F, agrarian A and modern M using a Kurzweilian information processing rate, $\dot{I}$, and Chaisson's specific power metric, $\dot{E}$. (Chaisson uses $\phi$ rather than $\dot{E}$ to represent specific power, which has units of W/kg.) Each of the three submatrices denotes a segment of humanity that is either above or below the average information processing average for that era, $\dot{\bar{I}}$, and above or below the average specific energy throughput, $\dot{\bar{E}}$. For example, the upper left corner of the matrix represents a society or a segment of society that processes an above-average amount of information, but a below-average amount of energy, whereas the lower right corner represents a society or a segment of society that is big on energy consumption, but low on information processing. Moreover, as explained by Diamond [28], any society living in the upper right corner of the matrix will either intentionally or unintentionally extinguish a society living in the lower left corner of the matrix, thus $\dot{\bar{I}}_{M}>\dot{\bar{I}}_{A}>\dot{\bar{I}}_{F}$ and $\dot{\bar{E}}_{M}>\dot{\bar{E}}_{A}>\dot{\bar{E}}_{F}$.

process energy (large $\dot{E}$ ), such as warriors, or otherwise large and violent individuals, etc. were likely more successful in producing offspring. So were those with larger information processing capabilities (large $I$ ), i.e. those with superior linguistic and abstraction skills. We thus subdivide individuals or individual societies from each Era into 'above average' and 'below average' categories on the basis of both of these two metrics (Fig. 3).

\section{CONCLUDING REMARKS}

Just as Christian divided the amount of time in each of the three Eras on two metrics: number of lives lived and number of years lived, we can further probe this for number of bits processed and amount of energy processed. The numbers become quite astonishing. Considering the Kurzweil metrics of $\dot{I}$ 
for humans as and the Chaissonian metrics for $\dot{E}_{\mathrm{m}}$, reasonable estimates are that less than $1 \%$ of information processed by humanity was processed during $\mathbf{F}$, less than $10 \%$ during $\mathbf{A}$ and more than $90 \%$ during M. In fact, with the surveillance occurring now, we can argue that a certain fraction of today's humans are living in the immediate present with their contemporaries, tracking their trajectories and extinguishing them far more effectively than would have been possible with the espionage tactics of previous ages. This is because the drone has an information processing rate of at least $10^{10}$ bits per second and an energy consumption rate of approximately $10^{5} \mathrm{~W}$ and thus $a_{\text {drone }}=3.73 \times 10^{7} \mathrm{~b} \mathrm{~K} \mathrm{~J}^{-1}$, which is on the order of that of the Google car. The information collected by the drone is then transmitted, processed and a binary decision is made whether or not to fully entropicize a particular individual thus removing him from Humanity, the socioeconomic realm, as well as and the future of 'Big History'.

As we move forward into the future, if we as a species are to remain successful and if we are to move towards a global society where fewer people are victimized and where the planet's resources are harvested in a sustainable manner, it seems critical that all people have better access to information. More importantly, it is critical that we open our eyes to information systems built upon deceit. We must also continue to develop information systems that allow us to monitor and begin to rein in our entropy production rate while somehow avoiding the fates of Tainter's societies so that we can avoid an anthropogenically driven collapse of the beautifully complex planet we have inherited. In conclusion, we turn to Kelvin, who stated:

When you can measure what you are speaking about, and express it in numbers, you know something about it; but when you cannot measure it, when you cannot express it in numbers, your knowledge is a meagre and unsatisfactory kind; it may be the beginning of knowledge, but you have scarcely, in your thoughts, advanced to the stage of science.

The author would therefore like to embolden the reader to refute McShea [43] who suggests abandoning words like complexity, information, entropy, etc. and rather canonize them in the same manner that the terms 'work', 'power', 'energy', 'stress' and 'strain' have been canonized in the engineering disciplines. The physical sciences and information sciences should follow suit.

In future work, the authors will explore the general observation that the second law typically involves the 'leaking' of energy from the direction of work as expressed by the mechanical form of the first law: $\Delta U=\Delta Q-\mathbf{F} \cdot \mathbf{d}$, where $U$ is the internal energy of the system, $Q$ is the system's thermal energy, and $\mathbf{F}$ and $\mathbf{d}$ are the force and displacement vectors operating on the mass in a specified direction, whereas heat will escape along the path of least resistance in all directions. Information transfer acts in a similar manner when portions of a message are lost to noise. Monetary taxes, the time value of money and other unpredictable factors such as the weather represent a similar 'friction' in financial transactions. Our next steps will be to demonstrate that the triad of energy, information and money all possess 'inefficiencies' when considered as inner products of multidimensional quantities in conjunction with the relationship: $N_{\text {states }}=N_{\text {reversible }}+1$.

\section{ACKNOWLEDGEMENTS}

The authors thank Eric Chaisson, Dick Coren and Russ Genet for their fruitful conversations on the topics of entropy and information. The authors declare no conflict of interest. This work was supported in part by the National Science Foundation DUE-1400670. Any opinions, findings and conclusions or recommendations expressed in this material are those of the authors and do not necessarily reflect the views of the National Science Foundation. 


\section{REFERENCES}

[1] Layton, B.E., Anthropogenic entropy acceleration and its relationship to Shannon information in the context of socioeconomics. Energy and Sustainability, 2014. WIT Transactions on Ecology and the Environment, 186, 2014. doi: http://dx.doi.org/10.2495/esus140271

[2] Feynman, R.P., Leighton, R.B. \& Sands, M.L., The Feynman Lectures on Physics, Vol. 2, Addison-Wesley: Reading, MA, 1963.

[3] Clausius, R., The Mechanical Theory of Heat, MacMillan: London, 1879.

[4] Carnot, S.N.L., Reflections on the Motive Power of Heat and on Machines Fitted to Develop that Power, John Wiley: New York, 1890.

[5] Chaisson, E.J., Energy rate density. II. Probing further a new complexity metric. Complexity, 17(1), pp. 44-63, 2011. doi: http://dx.doi.org/10.1002/cplx.20373

[6] Chaisson, E.J., Energy rate density as a complexity metric and evolutionary driver. Complexity, 16(3), pp. 27-40, 2011. doi: http://dx.doi.org/10.1002/cplx.20323

[7] Everett, R., et al., Energy Systems and Sustainability: Power for a Sustainable Future, Oxford University Press, 2012.

[8] Rolle, K.C., Thermodynamics and Heat Power, 6th edn., Pearson/Prentice Hall: Upper Saddle River, NJ, 2005.

[9] Worldometers. Worldometers Real Time World Statistics, , available at http://www.worldometers.info/, 7 March 2015.

[10] Layton, B.E., Dr. Bradley Layton April Energy Series 3 - Uncle Helios: A Financial Analogy to Global Fossil Fuel Consumption Rates, available at https://www.youtube.com/playlist?list= PLNM8cJC1mnW42dHuvto2K_5YlWN-rGrqe, 2015.

[11] Gal-Or, B., Cosmology, Physics, and Philosophy, Springer-Verlag: New York, 1983.

[12] SchröDinger, E., What is Life? The Physical Aspect of the Living Cell; With, Mind and Matter; \& Autobiographical Sketches, Cambridge University Press: Cambridge, New York, 2012.

[13] Lindeburg, M.R., Mechanical Engineering Reference Manual for the PE Exam, 12th edn., Professional Publications: Belmont, CA, 2006.

[14] Shankaranarayanan, S., Corrections to Bekenstein-Hawking entropy - quantum or not-so quantum? Entropy, 13, pp. 11-16, 2011. doi: http://dx.doi.org/10.3390/e13010011

[15] Ferhat, S. \& Fitzgerald, D., Comfort in courtyard house, with cool winter, 1988. 13th National Passive Solar Conference of the American Solar Energy Society, Massachusetts Institute of Technology, USA, 1988.

[16] Raman, A.P., et al., Passive radiative cooling below ambient air temperature under direct sunlight. Nature, 515, pp. 540-544, 2014. doi: http://dx.doi.org/10.1038/nature13883

[17] Jaynes, E.T., Gibbs vs Boltzmann entropies. American Journal of Physics, 33(5), pp. 391-398, 1965. doi: http://dx.doi.org/10.1119/1.1971557

[18] Tainter, J., Collapse of Complex Societies, available at https://www.youtube.com/ watch?v=G0R09YzyuCI, 2012.

[19] Hollifield, M., Thompson Fullilove, M. \& Hobfoll, S.E., Climate change refugees. Climate Change and Human Well-Being International and Cultural Psychology, pp. 135-162, 2011. doi: http://dx.doi.org/10.1007/978-1-4419-9742-5_8

[20] Sachs, J., Climate change refugees. Scientific American, 296, pp. 43, 2007. doi: http://dx.doi. org/10.1038/scientificamerican0607-43

[21] Shannon, C.E., et al., Claude Elwood Shannon: Collected Papers, IEEE Press: New York, 1993.

[22] Shannon, C.E., A mathematical theory of communication. Bell System Technical Journal, 27, pp. 379-423 \& 623-656, 1948. doi: http://dx.doi.org/10.1002/j.1538-7305.1948.tb00917.x

[23] Lin, S.-K., Diversity and entropy. Entropy, 1, pp. 1-3, 1999. doi: http://dx.doi.org/10.3390/ e1010001 
[24] Layton, B., The role of mechanoevolution in predicting the future of micro- and nanoscale technologies. Systems Engineering for Microscale and Nanoscale Technologies, eds. M.A.G. Darrin \& J. Barth, CRC Press: Boca Raton, FL, 2011.

[25] Layton, B.E. The application of game theory to thermoeconomics. Proceedings of the ASME 2011 International Mechanical Engineering Congress and Exhibition, 11-17 November 2011, Denver, CO.

[26] Layton, B.E., Mechanoevolution: an examination of the coevolution of humans and technology. Inaugural Meeting of the International Big History Association, 5 August 2012, Grand Rapids, MI, USA.

[27] Layton, B.E., Mechanoevolution: the possibility of deeper connections between information theory and the second law. ASME International Mechanical Engineering Congress and Exposition, 12-18 November 2010, Vancouver, BC, Canada.

[28] Diamond, J.M., Guns, Germs, and Steel : The Fates of Human Societies, 1st edn, W.W. Norton \& Co.: New York, 25, 1997.

[29] Layton, B.E., Recent patents in bionanotechnologies: nanolithography, bionanocomposites, cell-based computing and entropy production. Recent Patents in Nanotechnology, 2(2), pp. 72-83, 2008. doi: http://dx.doi.org/10.2174/187221008784534532

[30] Google, 2012. 8 Columbine Rd, available at https://www.google.com/maps/@46.894235,113.959996,3a,75y,277.68h,90t/data=!3m4!1e1!3m2!1sKOuDKh56KXbPEyZSW0AU1A!2e 0 ! $6 \mathrm{~m} 1$ ! $1 \mathrm{e} 1$

[31] Layton, B.E., A comparison of energy densities of prevalent energy sources in units of joules per cubic meter. International Journal of Green Energy, 5, pp. 438-455, 2008. doi: http:// dx.doi.org/10.1080/15435070802498036

[32] Prigogine, I., The Behavior of Matter Under Nonequilibrium Conditions: Fundamental Aspects and Applications, Center for Studies in Statistical Mechanics: Austin, TX, 1990.

[33] Genet, R., The Chimpanzees Who Would Be Ants: The Evolutionary Epic of Humanity, Nova Science Publications: Commack, NY, 1997.

[34] Genet, R.M., Personal Communication, 05 August 2012, Director, Inaugural Meeting of the International Big History Association, Orion Institute, Santa Margarita, CA.

[35] Dawkins, R., The Ancestor's Tale : A Pilgrimage to the Dawn of Evolution, Houghton Mifflin: Boston, MA, 2004.

[36] Dick, S.J. \& Lupisella, M., Cosmos \& Culture : Cultural Evolution in a Cosmic Context, Nasa $\mathrm{Sp}$, National Aeronautics and Space Administration, Office of External Relations for sale by the Supt. of Docs., U.S. G.P.O.: Washington, DC, 2009.

[37] Toffler, A., The Third Wave, 1st edn, Morrow: New York, 1980.

[38] Christian, D., David Christian: the history of our world in 18 minutes, available at http://www. ted.com/talks/david_christian_big_history.html, 2011.

[39] Nash, J., The bargaining problem. Econometrica, 18(2), pp. 155-162, 1950. doi: http://dx.doi. org/10.2307/1907266

[40] Wright, R., The Moral Animal : The New Science of Evolutionary Psychology, 1st edn, Pantheon Books: New York, 1994.

[41] Beinhocker, E.D., The Origin of Wealth: Evolution, Complexity, and the Radical Remaking of Economics, Harvard Business School Press: Boston, MA, 2006.

[42] Brandenburg, J. \& Rix Paxson, M., Dead Mars, Dying Earth/by John E. Brandenburg and Monica Rix Paxson, Crossing Press: Freedom, CA, 1999.

[43] Mcshea, D.W., Unnecessary complexity. Science, 342(13 Dec), pp. 1319-1320, 2013. doi: http://dx.doi.org/10.1126/science. 1245386 